\title{
Assessment of craniofacial hyperhidrosis and flushing by sphenopalatine blockade - a randomized trial*
}

\author{
Eduardo Lehrer, , Anna Nogués 1,2, Francesca Jaume4, Joaquim Mullol 1,2,3, , \\ Isam Alobid ${ }^{1,2,3,5, \#}$ \\ 'Rhinology and Skull Base Unit, Department of Otorhinolaryngology, Hospital Clinic, Universitat de Barcelona, Barcelona, \\ Catalonia, Spain \\ ${ }^{2}$ Institut d'Investigacions Biomèdiques August Pi i Sunyer (IDIBAPS), Barcelona, Catalonia, Spain \\ ${ }^{3}$ Centro de Investigación Biomédica en Red de Enfermedades Respiratorias (CIBERES), Barcelona, Catalonia, Spain \\ ${ }^{4}$ Department of Otorhinolaryngology, Hospital Comarcal d'Inca, Mallorca, Spain \\ ${ }^{5}$ Unidad Alergo Rino, Centro Médico Teknon, Barcelona, Catalonia, Spain
}

Rhinology 57: 1, 51 - 58, 2019

https://doi.org/10.4193/Rhin19.119

*Received for publication:

March 14, 2019

Accepted: July 12, 2019

\# these two authors shared senior

responsibilities

\begin{abstract}
Background: Craniofacial hyperhidrosis (CFH) and flushing express nervous system autonomic dysfunction. Available reference treatments lack good compliance. The study objective was to investigate variations of $\mathrm{CFH} /$ flushing after two methods of sphenopalatine ganglion (SPG) blockade.
\end{abstract}

Methodology: $\mathrm{CFH}$ patients ( $\mathrm{n}=25$ ) were randomized in a ratio of 1:3 in two groups; 1 ) endoscopic application of topical lidocaine over SPG (TL; n=7); 2) endoscopic injection of lidocaine in the SPG (IL; $\mathrm{n=18})$. CFH, flushing, rhinorrhoea, nasal obstruction, and smell detection were scored by Visual Analogue Scale (VAS). Nasal endoscopy, acoustic rhinometry, mucociliary transport test, smell/taste test, Schirmer test, Short Form-12, Chronic Skin Diseases Questionnaire, and Skin Satisfaction Questionnaire were also performed at visit $0,1,3$ and 6 months.

Results: At baseline, groups reported similar CFH VAS (TL: $89.3 \pm 17.5 \mathrm{~mm}$; IL: $85.7 \pm 22.1 \mathrm{~mm}$ ) or flushing VAS (TL: $52.7 \pm 30 \mathrm{~mm}$; IL: $59 \pm 33.8 \mathrm{~mm}$ ). After 6 months, the least squares mean of CFH VAS in IL was -38.1 (-47.3 to -28.9 ) compared to TL 1.9 (-12.2 to 15.9). However, flushing VAS did not improve. Any rhinological measure nor quality of life test showed significant changes. One patient presented controlled epistaxis intraoperatively during IL.

Conclusions: This preliminary study shows the sphenopalatine blockade injection as a safe procedure. Patients with CFH or flushing had significant improvement after lidocaine injection which lasted 6 months. Due to the small sample and the lack of objective measures more studies are needed.

Key words: autonomic nervous system, flushing, craniofacial hyperhidrosis, sphenopalatine ganglion blockade, nasal endoscopy

\section{Introduction}

Autonomic dysfunction of the head and neck is mainly represented by idiopathic craniofacial hyperhidrosis (CFH) and flushing. $\mathrm{CFH}$, which is a primary focal hyperhidrosis, consists of secretion of excessive sweat in greater amounts than physiologically necessary for thermoregulation that can lead to serious psychosocial consequences. It represents a $10 \%$ of all primary hyperhidrosis that has an overall incidence of $1-2.8 \%$ in general population ${ }^{(1)}$. Flushing refers to a transient erythema of the face associated to a sensation of warmth as a consequence of an increased cutaneous blood flow secondary to vasodilation ${ }^{(2)}$. Its prevalence is unknown, but social phobia, which is a psychiatric pathology linked to flushing rounds $13 \%{ }^{(3,4)}$. The pathophysiology of idiopathic $\mathrm{CFH}$ and flushing is poorly understood but it seems to share an autonomic hyperreflexia as a response to normal situations or emotional stress ${ }^{(5)}$.

Having this theory as background, the sphenopalatine ganglion (SPG) could play a role in the pathology as site of this autonomic 
disfunction. The SPG lies in the pterygopalatine fossa behind the posterior wall of the maxillary sinus and lateral to the perpendicular plate of the palatine bone. Prassanna and Murthy in 1993 reported a case in which a topical blockade of SPG with $4 \%$ xylocaine for 5 minutes in two sessions was performed and diminished a recalcitrant $\mathrm{CFH}$ on a patient who previously tried a variety of treatments ${ }^{(6)}$. We hypothesized that SPG blockade would be effective for controlling $\mathrm{CFH}$ and flushing. In this sense, a blockade technique is proposed by endonasal endoscopic numbing of the SPG area. This blockade would be a fast, safe and well-accepted technique to be done in an outpatient clinic. $\mathrm{CFH}$ and flushing are symptoms that could be seen in other known conditions more severe, such as familial dysautonomia, however they would show not only in one body surface and would associate sensitive manifestations among other symptoms ${ }^{(7)}$. A consensus statement in $2004^{\left({ }^{(8)}\right.}$ presented the diagnostic criteria for idiopathic hyperhidrosis. In terms of flushing, once other causes are discarded (neurologic disorders, concomitant medication, alcohol abuse, carcinoid syndrome, mastocytosis, pheochromocytoma) ${ }^{(9)}$, it may be considered as idiopathic or related to a specific autonomous mediated cause (e.g. fever, hyperthermia, menopause, emotional blushing). Quantitative and qualitative methods for sweating measurements, with their strengths and pitfalls, have been developed such as gravimeter, the Minor iodine-starch test, the Hyperhidrosis Disease Severity Scale (HDSS) ${ }^{(10)}$, and Patient Reported Outcomes (PROs) by visual analogue scale (VAS).

In terms of treatment, the exposure area of CFH and flushing is often considered as a limiting factor for some of the available therapies. The major treatment options start with topical therapies and then try systemic drugs, botulinum toxin injections, and thoracoscopic surgery. Nevertheless, data on these treatments specifically for the area of interest are limited. None of these available techniques are free from side effects. Transthoracic sympathectomy, which is an invasive procedure directed to section the thoracic sympathetic ganglionar chain, is the most published procedure to treat these conditions. The results differ however in the level of satisfaction and quality of life (QoL) in relation to the location of the symptoms and techniques ${ }^{(11)}$. The main aims of this study were to measure the efficacy of blocking SPG hyperreactivity using two different techniques, either by a direct injection of the ganglion area or by applying topical anaesthesia. Additional objectives were to rule out the safety of these procedures and to assess the impact on patient's QoL.

\section{Materials and methods}

\section{Study design}

This study was a randomized, but unblinded trial on patients with primary CFH or flushing. Individuals were recruited from June 2013 to June 2014 at the hyperhidrosis unit of a third level referral hospital. All patients were randomly allocated to two treatment groups: 1) endoscopic application of topical lidocaine on the site of SPG (TL group); 2) endoscopic injection of lidocaine on the site of SPG (IL group). Both procedures were performed by the same surgeon (EL) and in the same environment conditions in order to avoid background biases. The trial was approved by our Institutional Ethics Committee (No HCB/2013/8365) and all patients provided their informed consent.

\section{Study population}

A total of 65 patients ( 58 with CFH and 7 with flushing) were included in the study. Primary focal hyperhidrosis is considered when there is focal, visible, excessive sweating of at least 6 months duration without apparent cause with at least two of the following characteristics: bilateral and relatively symmetric, impairing of daily activities, frequency of at least one episode per week, age of onset less than 25 years, positive family history, or cessation of focal sweating during sleep ${ }^{(8)}$.

All patients with CFH had moderate to severe disease according to levels 3 or 4 of HDSS. Each patient before get included in the trial tried topical applications of aluminium chloride and there were some cases of skin stains. Furthermore, topical ointments resulted uncomfortable. They also proved available anticholinergic medication such as solifenacin and oxybutynin but systemic side effects were frequently found and there was no good compliance. Nasal computed tomography was performed in order to discard any anatomical variations or secondary sinuses inflammatory disease. Patients younger than 18 years-old, with systemic or multifocal hyperhidrosis, such as familial dysautonomia, menopausal flushes with no history of primary focal hyperhidrosis, severe septal deviation, chronic rhinosinusitis with/without nasal polyps, or nasal malignancies were excluded. Only 28 patients older than 18 years met the inclusion criteria and then were randomized in a ratio of 1:3 for TL or IL respectively.

\section{Therapeutic techniques}

All patients underwent in supine position with the same room conditions. In the TL group, the application of intranasal 4\% lidocaine was done bilaterally with cottonoids for 15 minutes. However, patients in IL group were bilaterally injected with $4 \%$ lidocaine by lumbar needle (22G) after a previous topical lidocaine with cottonoids (for about 5 minutes). SPG blockade was done at the level of the attachment of the middle turbinate to the medial plate of the pterygopalatine process (Figure 1).

\section{Study outcomes}

All study measurements were performed after a short period of acclimatization to minimize the effects of physical stress and temperature changes at baseline and visit 1, 3, and 6 months. 


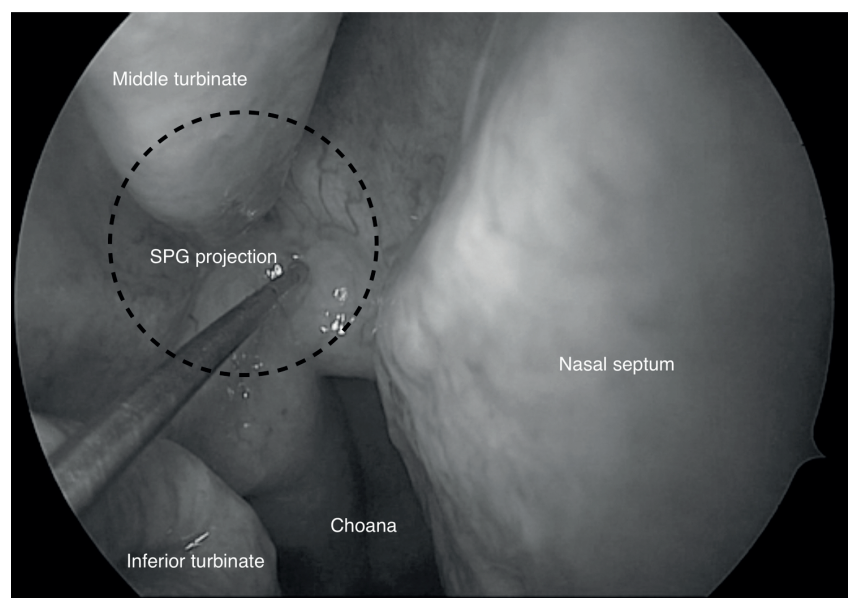

Figure 1. Injection site of the sphenopalatine ganglion (SPG) through nasal endoscopy of the right nasal fossa. Endoscopic view $\left(0^{\circ}\right)$ of the area of "SPG projection".

\section{A) Subjective outcomes}

- PROs. CFH, flushing, rhinorrhoea, nasal obstruction, and smell detection are scored by VAS (0-100 $\mathrm{mm})$.

- Olfactometry and gustometry. Barcelona Smell Test 24 (BAST24) was used to measure the sense of smell. BAST-24 consists of 24 odours, in which 20 odours assess the 1st cranial nerve (olfactory): banana, gasoline, lemon, rose, onion, smoked, anise, coconut, vanilla, melon, mandarin, bitter almond, pineapple, cheese, strawberry, mushroom, eucalyptol, clove, turpentine, and peach; and 4 odours asses the 5 th cranial nerve (trigeminal): formaldehyde, vinegar, ammonia, and mustard. After being exposed for 5 seconds to odours, patients were asked in order to score by means of percentage (\%) smell detection (Did you smell anything?), identification/ olfactory recognition/memory (Did you recognize this odour?), and forced choice (Which of these four odours did you smell?) ${ }^{(12)}$. Gustometry was also assessed after letting the patient to try salt, sweet, acid, bitter, and umami and then asking a forced choice question (Which of these five tastes did you try?) in order to get a percentage of guess.

- Quality of Life questionnaires. Three instruments were used in the trial. One of them was generic (SF-12) while the other two were specific for skin conditions.

1. Short Form-12 (SF-12) Health Survey. This questionnaire consists of 12 self-administered questions that cover eight health domains grouped into two summaries: 1) physical summary includes physical functioning, role physical, bodily pain, and general health; and 2) mental summary includes vitality, role emotional, social functioning, and mental health. Scale scores range from 0 to 100 and higher scores indicate better $\mathrm{QoL}^{(13)}$.

2. Chronic Skin Diseases Questionnaire (ACS). This was developed to assess problems related to chronic skin disorders. It comprises 51 questions grouped in 6 subscales about social anxiety/ avoidance, itch-scratch cycle, helplessness, anxious-depressive mood, impact on QoL, and deficit in active coping. Higher scores reflect more negative impact on specific $Q \mathrm{QL}^{(14)}$.

3. Skin Satisfaction Questionnaire (SSQ). It was created to assess satisfaction toward self-skin in patients with dermatologic problems as in healthy subjects. SSQ assesses a broad range of parameters on specific psychosocial aspects of skin perception and satisfaction in 5 areas: partnership touching, shame, family touching, disgust, and self-touching. Higher scores reflect more negative impact on specific QoL ${ }^{(15)}$.

\section{B) Objective outcomes}

- Sinonasal findings. Nasal endoscopy was performed through a rigid $0^{\circ}$ nasal endoscope before and after procedures at every visit in order to rule out previous pathologies (such as rhinitis, septal deformities, nasal polyposis) and then look for changes (such as, changes in colour and aspect of the mucosa of inferior and middle turbinates).

- Acoustic rhinometry (AcR). An Acoustic rhinometer SER 2000 (RhinoMetrics, Lynge, Denmark) was used. All AcR measurements were repeated three times in order to ensure the reproducibility of the results. The default software settings were used for the parameters of most interest, which were the minimum cross-sectional area (mCSA) and nasal cavity volume from 0 to 6 $\mathrm{cm}$ (vol0-6) from the end of the nose piece. Repeated measurements were performed and the mean of three values were recorded for mCSA and vol0-6 $6^{(16)}$.

- Mucociliary transport test (MCT). MCT is a simple, noninvasive, and economic method that yields reproducible results. Patients were told not to bend forward and not to sniff, inhale or exhale forcefully while avoiding nasal manipulation during the test. At first, nasal secretions were aspirated and then a particle of saccharine ( $1 \times 1 \times 1 \mathrm{~mm}$ size) was placed on the floor of the nose $1 \mathrm{~cm}$ posterior to the anterior end of inferior turbinate. The time in seconds required by the individual to perceive the sweet taste was noted. Values took as normal were up to 20 minutes $^{(17)}$. Patients were asked to report any change in taste and were not told that they would perceive a sweet taste.

- Schirmer test. It is a common test for quantitatively measuring tearing. The test consists of a standard strip of filter paper placed on both eyes over the margin of lower eyelids for five minutes with the eyes gently closed. Wetting filter paper less than $5 \mathrm{~mm}$ demonstrates dry eye. Findings are usually seen in a symmetrical way ${ }^{(18)}$.

\section{Statistical analysis}

The sample size was calculated to have $80 \%$ power to detect a difference of at least $20 \%$ between both groups in terms of improvement in CFH 6 months after application of $4 \%$ lidocaine. 25 patients were needed in total to detect significant differences. Descriptive analyses were applied for demographics, and baseline characteristics. A mixed-effect model with repeated 


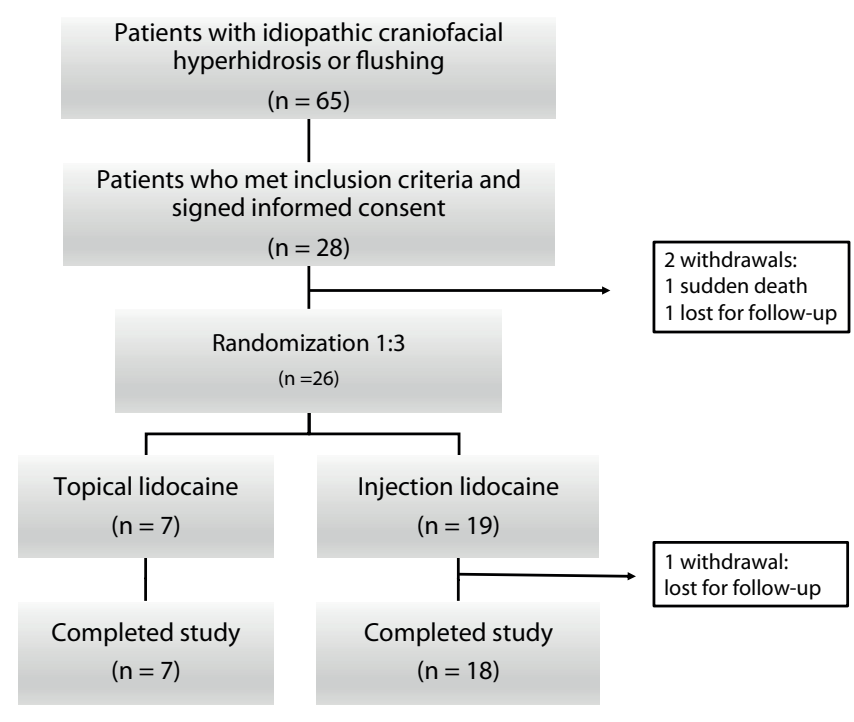

Figure 2. Flow diagram of craniofacial hyperhidrosis and flushing patients included in the study.
Table 1. Demographic characteristics of both experimental groups.

\begin{tabular}{|c|c|c|c|}
\hline Characteristics & $\begin{array}{c}\text { Topical } \\
\text { lidocaine } \\
n=7\end{array}$ & $\begin{array}{l}\text { Injection } \\
\text { lidocaine } \\
n=18\end{array}$ & p-value \\
\hline Female, n (\%) & $6(85.7)$ & $15(83.3)$ & 1.000 \\
\hline Age (years), mean (SD) & $60.7(6.7)$ & $52.8(14.4)$ & 0.074 \\
\hline Diagnosis & & & 1.000 \\
\hline $\begin{array}{l}\text { Hyperhidrosis w/o } \\
\text { flushing, } \mathrm{n}(\%)\end{array}$ & $7(100)$ & $16(88.9)$ & \\
\hline $\begin{array}{l}\text { Isolated flushing, } \\
\mathrm{n}(\%)\end{array}$ & $0(0)$ & $2(11.1)$ & \\
\hline $\begin{array}{l}\text { Family history of CFH } \\
\text { or flushing, } \mathrm{n}(\%)\end{array}$ & $5(71.4)$ & $13(72.2)$ & 1.000 \\
\hline $\begin{array}{l}\text { Body mass index, } \\
\text { mean (SD) }\end{array}$ & $30.0(4.9)$ & $28.9(4.5)$ & 0.657 \\
\hline
\end{tabular}

SD, standard deviation; CFH, craniofacial hyperhidrosis. measures (MMRM) was the approach to analyse data of the study population. This model included the change of VAS in terms of CFH and flushing in both groups, that of topical lidocaine and the other of injected lidocaine from baseline to follow-up time. We included in the model baseline value, treatment, time and treatment time interaction; with restricted maximum likelihood approach of estimation. We used 95\% confidence intervals and Tukey-adjusted comparisons from MMRM in order to compare treatment effects at each time.

Figures of outcomes variables were expressed as least squares mean (LMS) change. There were used two-sample Student's t-test for a mean ratio with a coefficient of variation of 1 , setting alpha at 0.05 ( $p$-value). The analysis of the data was executed using the program SPSS ${ }^{\circledR} 23.0$ statistical software package (SPSS, Chicago, IL, USA).

\section{Results}

A total of 21 patients were randomly assigned to the IL group and 7 to the TL group (Figure 2). In the IL group, one patient presented a sudden cardiac arrest at home before the procedure, one patient did not show up in the visit day, and 1 patient did not follow any postoperative control. Finally, only 18 patients completed the study in the IL group. There were no differences between $T L$ and IL groups regarding gender, age, family history or body mass index (Table 1).

\section{Subjective outcomes}

- $\mathrm{CFH}$

At baseline, patients in both TL and IL groups had similar CFH severity by VAS ( $89.3 \pm 17.5 \mathrm{~mm}$ vs $85.7 \pm 22.1 \mathrm{~mm}$ respectively). The LMS change between baseline and 1 month was for TL -0.3
(95\% Cl, -14.3 to 13.7$)$ and for IL $-57.6(95 \% \mathrm{Cl},-66.5$ to -48.6$)$, $(p<0.001)$. This steep slope of CFH was observed in IL-treated group compared to the TL group at 3 and 6 months respectively (Figure 3).

\section{- Flushing}

At baseline, patients in both TL and IL groups had similar flushing severity by VAS $(52.7 \pm 30 \mathrm{~mm}$ vs $59 \pm 33.8 \mathrm{~mm}$, respectively). After TL, no patient reported an improvement on flushing (1-month, p 0.092). Patients from the IL group showed changes in the LMS but without reaching statistical significance (Figure 4).

\section{- PROs by VAS}

At baseline, both groups showed similar VAS levels on rhinorrhea or smell detection without any improvement after TL or IL. None of these variables evidenced steady and significant changes in time (Table 2).

\section{- Quality of Life}

SF-12. No significant differences were reported at baseline comparing TL and IL groups. The follow-up on SF-12 did not show any significant improvement after SPG therapy. No differences were observed between TL and IL procedures after 1, 3 or 6 months (Figure 5).

Skin Satisfaction Questionnaire (SSQ). There were no significant differences on SSQ at baseline between TL (83.5 \pm 7.34$)$ and IL $(80.8 \pm 12.5)$. Adjusted mean results of SSQ at 1,3 and 6 months did not reveal changes during the trial (data not shown). None of the subscales neither showed significant differences among groups. 


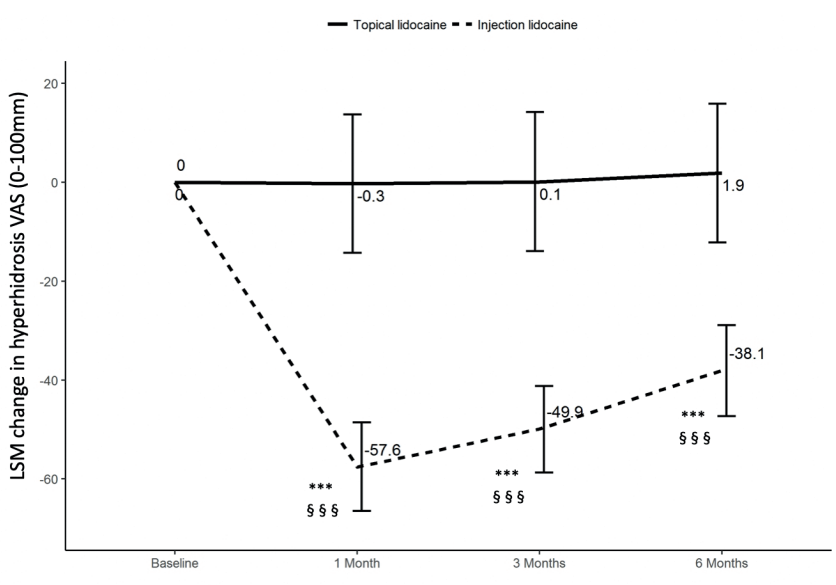

Figure 3. Visual Analogue Scale (VAS, 0-100mm) of craniofacial hyperhidrosis after topical or injected lidocaine ( $\mathrm{CFH}, \mathrm{n}=25)$. Data are presented as the least squares mean change (LSM) and standard error of the mean (SEM). Two-sample Student's t-test. ${ }^{\$ \varsigma 5}, \mathrm{p}<0.001$ compared to baseline; *** $p<0.0001$ compared with topical lidocaine group.

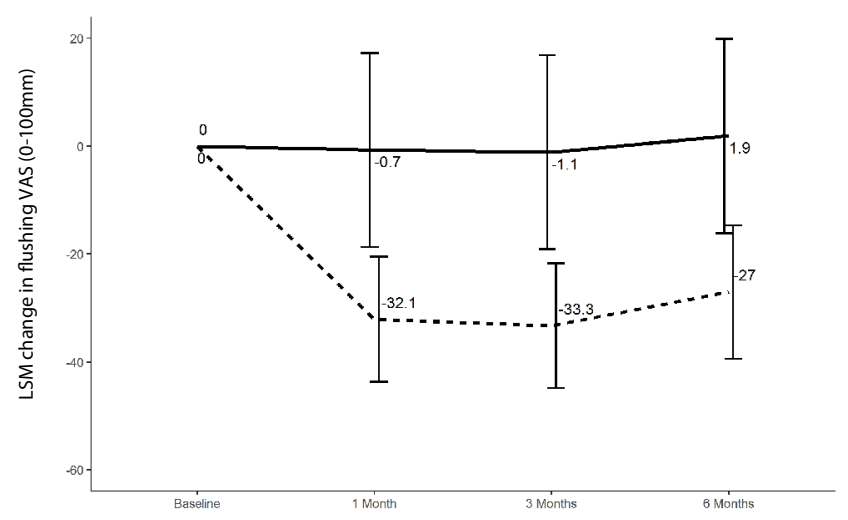

Figure 4. Flushing Visual Analogue Scale (VAS, 0-100mm) after topical or injected lidocaine in patients with craniofacial hyperhidrosis (CFH, $\mathrm{n}=25$ ). Data are presented as the least squares mean change (LSM) and standard error of the mean (SEM). Two-sample Student's t-test. No evidence of significance.

Chronic Skin Diseases Questionnaire (ACS). There were no significant differences on ACS at baseline between TL $(175 \pm 22)$ and IL (152 \pm 37.7$)$. ACS did not show any changes after 1, 3 and 6 months in any of its subscales in any group.

\section{Objective outcomes}

- Measurement of AcR, MCT, olfactometry, gustometry, and Schirmer test. Both groups showed similar scores at baseline, and after 1, 3, and 6 months of treatment without any differences between TL and IL groups (Table 3, 4). No improvement was achieved in any of these outcomes.

- Safety. Both groups, TL and IL, were advised to notify any symptom in the 2 following weeks after the blockade. None of
Table 2. Measurement of sinonasal symptoms by Visual Analogue Scale (VAS, $0-100 \mathrm{~cm})$.

\begin{tabular}{|c|c|c|c|}
\hline & $\begin{array}{c}\text { Topical } \\
\text { lidocaine } n=7\end{array}$ & $\begin{array}{c}\text { Injection } \\
\text { lidocaine } n=18\end{array}$ & $\begin{array}{c}\text { p- } \\
\text { value }\end{array}$ \\
\hline \multicolumn{4}{|c|}{ Rhinorrhea, mean (SEM) } \\
\hline Baseline & $17.4(15.2)$ & $22.1(21.5)$ & 0.550 \\
\hline LSM, 1 month & $0.4(27.7)$ & $14.7(29.3)$ & 0.622 \\
\hline LSM, 3 months & $-0.3(27.7)$ & $6.8(17.2)$ & 0.982 \\
\hline LSM, 6 months & $0.9(27.7)$ & $-3(18.2)$ & 1.000 \\
\hline \multicolumn{4}{|c|}{ Nasal obstruction, mean (SEM) } \\
\hline Baseline & $57.0(28.7)$ & $30.4(30.4)$ & 0.064 \\
\hline LSM, 1 month & $-0.6(31.6)$ & $-5.6(20.3)$ & 0.999 \\
\hline LSM, 3 months & $-0.3(31.6)$ & $-14.3(28.3)$ & 0.767 \\
\hline LSM, 6 months & $-3.7(31.6)$ & $-8.4(20.9)$ & 0.999 \\
\hline
\end{tabular}

VAS, Visual Analog Scale; LSM, least squares mean change; SEM, standard error of the mean.

Table 3. Assessment of objective outcomes.

\begin{tabular}{|cccc|}
\hline Acoustic rhinometry & $\begin{array}{c}\text { Topical } \\
\text { lidocaine } \mathbf{n = 7}\end{array}$ & $\begin{array}{c}\text { Injection } \\
\text { lidocaine } \mathbf{n = 1 8}\end{array}$ & $\begin{array}{c}\mathbf{p -} \\
\text { value }\end{array}$ \\
\hline Baseline & $1.52(0.56)$ & $1.42(0.37)$ & 0.710 \\
\hline LSM, 1 month & $-0.4(1.3)$ & $0.3(0.6)$ & 0.69 \\
\hline LSM, 3 months & $-0.1(1.3)$ & $-0.2(0.8)$ & 1.000 \\
\hline LSM, 6 months & $-0.1(1.3)$ & $-0.2(0.8)$ & 1.000 \\
\hline
\end{tabular}

Acoustic rhinometry (vol 0-6), mean (SEM), $\mathrm{cm}^{3}$

\begin{tabular}{|rccc|}
\hline Baseline & $25.5(8.29)$ & $24.7(9.86)$ & 0.835 \\
\hline LSM, 1 month & $-2.3(12.5)$ & $0(7)$ & 0.997 \\
\hline LSM, 3 months & $2.2(12.5)$ & $6(7)$ & 0.944 \\
\hline LSM, 6 months & $2.3(12.4)$ & $3.8(7.6)$ & 1.000 \\
\hline
\end{tabular}

Mucociliary transport test mean (SEM), seconds

\begin{tabular}{|c|c|c|c|}
\hline Baseline & 1104 (535) & $893(578)$ & 0.443 \\
\hline LSM, 1 month & $-390.4(780.9)$ & $-282.5(565)$ & 0.997 \\
\hline LSM, 3 months & $-320.4(640.9)$ & $-306.8(613.7)$ & 1.000 \\
\hline LSM, 6 months & $-350.4(700.9)$ & $-347.3(694.5)$ & 1.000 \\
\hline \multicolumn{4}{|c|}{ Schirmer test mean (SEM), mm } \\
\hline Baseline & $10.7(4.88)$ & $17.3(9.58)$ & 0.046 \\
\hline LSM, 1 month & $0.3(9.9)$ & $4.7(9.3)$ & 0.768 \\
\hline LSM, 3 months & $1.7(9.9)$ & $3.6(7.3)$ & 0.996 \\
\hline LSM, 6 months & $1.3(9.9)$ & $2.7(6.3)$ & 1.000 \\
\hline
\end{tabular}

mCSA, minimal Cross-Sectional Area; vol 0-6, nasal cavity volume from 0 to $6 \mathrm{~cm}$; LSM, least squares mean change; SEM, standard error of the mean. 
Table 4. Assessment of subjective olfactometry (BAST-24) and gustometry.

\begin{tabular}{|c|c|c|c|}
\hline & $\begin{array}{c}\text { Topical } \\
\text { lidocaine } n=7\end{array}$ & $\begin{array}{c}\text { Injection } \\
\text { lidocaine } n=18\end{array}$ & $\begin{array}{c}\text { p- } \\
\text { value }\end{array}$ \\
\hline \multicolumn{4}{|c|}{ Smell detection \%, mean (SEM) } \\
\hline Baseline & $100(0)$ & $100(0)$ & 1.000 \\
\hline LSM, 1 month & $0(0)$ & $0(0)$ & 1.000 \\
\hline LSM, 3 months & $0(0)$ & $0(0)$ & 1.000 \\
\hline LSM, 6 months & $0(0)$ & $0(0)$ & 1.000 \\
\hline \multicolumn{4}{|c|}{ Smell recognition / memory \%, mean (SEM) } \\
\hline Baseline & $28.6(12.8)$ & $19.9(12.7)$ & 0.157 \\
\hline LSM, 1 month & $1.6(17.1)$ & $3.5(10.9)$ & 1.000 \\
\hline LSM, 3 months & $2.8(17.1)$ & $5.8(11.6)$ & 0.998 \\
\hline LSM, 6 months & $2.8(17.1)$ & $9.1(18.1)$ & 0.904 \\
\hline \multicolumn{4}{|c|}{ Smell identification forced-choice, \%, mean (SEM) } \\
\hline Baseline & $73.8(12.2)$ & $70.1(15.0)$ & 0.538 \\
\hline LSM, 1 month & $-4.3(23.6)$ & $-1.9(15.1)$ & 1.000 \\
\hline LSM, 3 months & $-13.2(26.4)$ & $-8.7(17.5)$ & 0.997 \\
\hline LSM, 6 months & $-13.2(26.4)$ & $-9.6(19.3)$ & 0.999 \\
\hline \multicolumn{4}{|c|}{ Taste identification \%, mean (SEM) } \\
\hline Baseline & $96.4(9.45)$ & $94.4(13.7)$ & 0.686 \\
\hline LSM, 1 month & $-0.2(13.2)$ & $4(8.3)$ & 0.947 \\
\hline LSM, 3 months & $3.4(13.1)$ & $-0.2(8.2)$ & 0.976 \\
\hline LSM, 6 months & $3.4(13.1)$ & $2.7(8.7)$ & 1.000 \\
\hline
\end{tabular}

BAST-24, Barcelona Smell Test 24 odours; LSM, least squares mean change; SEM, standard error of the mean.

TL patients presented any complication or side effect after the mentioned period. However, compared to TL, IL group showed few mild symptoms. During IL blockade, one patient had epistaxis that was totally controlled during the procedure and did not require nasal packing. Other 5 patients referred mild ocular irritation and dryness sensation (1/5); headache (1/5); or a combination of rhinorrhoea, headache, and ocular irritation
(3/5). Each of these adverse reactions were told to be minimal and solved in the first week after the blockade without any additional treatment.

\section{Discussion}

The main findings of this study were: 1st) patients with CFH and flushing showed significant improvement of CFH after lidocaine injection in SPG area which lasted at least for 6 months; and 2nd) no significant complications were found using this therapeutic procedure.

To our knowledge, this is the first randomized trial that reveals some effects in terms of regulation of $\mathrm{CFH}$ and flushing through direct action on SPG. Lidocaine injection of SPG probably acts by reducing the release of nitric oxide synthetase at the level of the node, thus reducing vascular response and minimizing sweating $^{(19)}$.

Prassanna and Murthy in 1993 reported a case in which the application of cotton swabs to the sphenopalatine area with $4 \%$ xylocaine for 5 minutes in two sessions diminished a recalcitrant facial hyperhidrosis on a patient who previously and unsuccessfully tried a variety of treatments ${ }^{(6)}$. Without providing the outcome measure for the patient's improvement, the authors reported that the patient improved in 50\% after the first application and in $90 \%$ after the second application. No publication about treating CFH in this way has been reported since then. To address this lack of research in that area few contributing factors can be considered. This is not a common disease seen by otolaryngologists who are more prone to assess and understand nasal anatomy and physiology; there is no consensus among different medical specialities to perform a multidisciplinary follow-up of these patients; the location of symptoms in the face and head constitutes a limiting factor for available therapies ${ }^{(20)}$; and the difficulty to properly diagnose this condition even when CFH and flushing negatively impact on QoL.
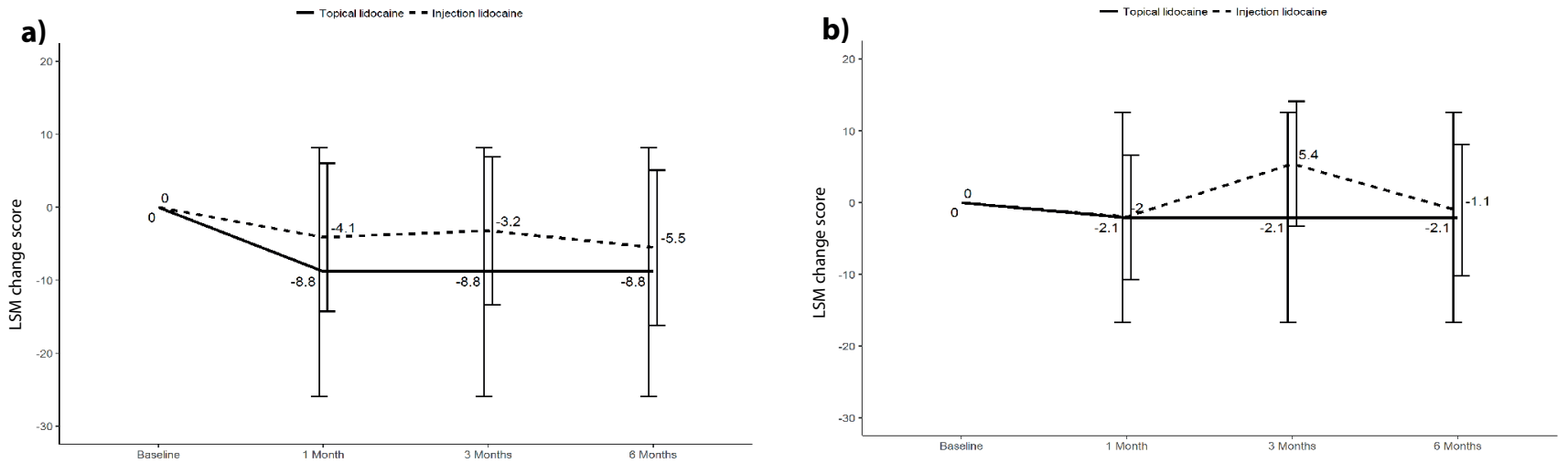

Figure 5. Impact of topical (TL) or injection (IL) of lidocaine in patient's quality of life by the Short Form-12 (SF-12). Data are presented as the least squares mean changes and standard error of the mean (SEM). Two-sample Student's t-test. No evidence of significance. 5a) SF-12 physical domain. 5b) SF-12 mental domain. 
All patients included in the trial were chosen from the Hyperhidrosis Unit of our hospital, mainly constituted by dermatologists and thoracic surgeons. Subjects in the trial were graded as 3 or 4 by the HDSS ${ }^{(10)}$ and they proved a variety of different treatments (topical aluminium chloride, oxybutynin, solifenacin) with unsatisfactory results before the screening and randomization process. The fact that every trial subject was a 'severe case' served as a homogenization element which provided us accord in the diagnosis and allowed us to prosecute in the evaluation of treatment.

Along with VAS, the study of QoL with PROs commonly used in rhinology were chosen as the best possible methods to assess changes between groups considering that known objective test such as Minor's iodine test or gravimetry show significant inter and intrapatient variability.

Both techniques compared in this study are easy to perform and reliable. IL performed transnasally has shown to be safe in other indications such as facial pain ${ }^{(21)}$, or as adjuvant procedure during endoscopic sinus surgery ${ }^{(22)}$. Unlike Prassanna and Murthy ${ }^{\left({ }^{6}\right)}$, in this study the topical application of anaesthesia to the SPG area did not reveal those reported changes. After directly injecting the SPG area improvement was however obtained. Although Prassanna and Murthy executed the topical blockade in two consecutive sessions, this trial has not evaluated the effect of a second IL or TL therapeutic session. We could however demonstrate a significant effect of the IL therapy with a minimum duration of 6 months.

The tangled topography of nerve fibres suggests a theoretical poor recovery after disruption of the fascicles, (i.e., trauma due to intraneural injections). In this sense, perineurium acts as a formidable barrier to lesions ${ }^{(23)}$. The membrane potential of neurons varies across different type of nerve fibres. The membrane is less permeable to sodium as compared to potassium. As a result, the potassium ions which diffuse inside are retained by the negatively charged intracellular proteins; hence they maintain a steep gradient resulting in a negative resting membrane potential. Local anaesthetics bind to specific sites at the inner pore of sodium channels clustered around the Nodes of Ranvier creating an electrostatic field that repels positively charged ions like sodium, thus blocking its action. Lidocaine reduces to a minimum the secretion of acetylcholine, due to the blockade of SPG preganglionic and, therefore, the release of nitric oxide synthetase is reduced as the consequent autonomic action.

IL performed transnasally is an outpatient procedure which has not revealed any sequel after the following-up period. The theoretical counter effect of compensatory hyperhidrosis after injection was not evidenced in our patients. Neither was observed any changes on nasal parameters such as rhinor- rhoea, nasal obstruction, smell, or taste. Compared to vidian neurectomy used for persistent allergic rhinitis ${ }^{(24)}$, IL results less harmful. Videoendoscopic thoracic sympathectomy indicated for flushing or upper body hyperhidrosis is not exempted of risks since subcutaneous emphysema, hemothorax or pneumothorax, Horner syndrome, and persistent local pain are among the frequent complications seen after this procedure. The usual need of pleural drainage is another matter to consider. Compensatory sweating among patients treated by thoracic sympathectomy rounds between 44 to $86 \%$ depending on the authors ${ }^{(25)}$. In comparison to alpha botulinum toxin injection directed to the sweating skin, IL therapy does not leave any transient weakness of facial musculature ${ }^{(26)}$. Topical application of anticholinergic agents, such as glycopyrrolate, implies a daily usage to control symptoms while having anticholinergic side effects ${ }^{(11)}$.

QoL questionnaires are useful for measuring changes after any procedure or treatment. In spite of this, special consideration is needed for those generic instruments that may be poorly scaled for disability research or could be misinterpreted ${ }^{(27)}$. In this study, the authors noticed that QoL was highly affected in the trial population. Aside, there was a high level of anxiety and distrust, possibly related to dissatisfaction after other unsuccessful therapeutic procedures for relieving the autonomic dysfunction. However, other psychological or emotional underlying conditions in these patients with recalcitrant symptoms cannot be discarded considering that their mean values on physical and mental health evaluation in the SF-12 were almost 50 points lower than values on general population.

Although the ACQ questionnaire ${ }^{(14)}$ was designed to evaluate QoL in patients with chronic skin conditions, we did not visualized changes between our two groups of treatment of CFH and flushing, either at basal status of after therapeutic evaluation. SSQ was designed for assessing specific psychosocial aspects of skin perception, skin satisfaction and attitudes toward the skin ${ }^{(15)}$. In the 'shame' subscale, patients showed comparatively differences between treatments that were maintained during the trial. In any case, results in terms of QoL in CFH and flushing must be further analysed because of the lack of specific instruments to rely on.

\section{Study limitations}

1st) The sample of patients with flushing was small. 2nd) HDSS was originally considered for selecting patients but the trial was not designed for detecting changes in its terms. 3rd) Additional objective methods for quantifying $\mathrm{CFH}$ and flushing were not employed. For instance, the Minor's test is useful in mapping areas of excessive sweating prior to treatment, but does not provide accurate information on the quantity of sweat produced. Glogau et al. ${ }^{(28)}$ suggested that focal sweating of more than 50 - 
100mg in 5 minutes measured by gravimetry can be considered as hyperhidrosis. Despite considerable efforts to achieve it, no standard cut-off value could be however applied to differentiate normal from pathologic sweating. In axillae, Bahmer developed the HASI-Hyperhidrosis Area and Secretion Index to quantify secretion in $\mathrm{mg} / \mathrm{cm}^{2}$ per minute by estimating the sweating area morphometrically after gravimetry and Minor iodine-starch test $^{(29)}$. Such approximation to objectively quantify the sweat at craniofacial area has not been developed and at least it could be challenging, because of the expected surface changes in the different areas of face and head, and the possibility of staining these sensible body parts.

\section{Acknowledgements}

Eduardo Lehrer was awarded with the Emili Letang Grant 20132014 to perform this research project.

\section{Authorship contribution}

EL: Total participation on the trial; AN: Participation during follow-up visits; FJ: Participation during follow-up visits; JM: Supervision and direction of the trial. IA: Supervision and direction of the trial.

\section{Conflict of interest}

Authors of this article have no conflicts of interest.

\section{References}

1. Nicholas R, Quddus A, Baker DM. Treatment of Primary Craniofacial Hyperhidrosis: A Systematic Review. Am J Clin Dermatol. 2015;16(5):361-70.

2. Wilkin JK. The red face: flushing disorders. Clin Dermatol. 1993;11(2):211-23.

3. Zamorski MA, Ward RK. Social anxiety disorder: common, disabling, and treatable. J Am Board Fam Pract. 2000;13(4):251-60.

4. Stein DJ, Ruscio AM, Lee $S$, et al. Subtyping social anxiety disorder in developed and developing countries. Depress Anxiety. 2010;27(4):390-403.

5. Izikson L, English JC, Zirwas MJ. The flushing patient: Differential diagnosis, workup, and treatment. J Am Acad Dermatol. 2006;55(2):193-208.

6. Prasanna A, Murthy PS. Sphenopalatine ganglion block under vision using rigid nasal sinuscope. Reg Anesth. 1993;18(2):139-40.

7. Morini E, Gao D, Montgomery CM, et al. ELP1 Splicing Correction Reverses Proprioceptive Sensory Loss in Familial Dysautonomia. Am J Hum Genet. 2019;113.

8. Hornberger J, Grimes K, Naumann M, et al. Recognition, diagnosis, and treatment of primary focal hyperhidrosis. J Am Acad Dermatol. 2004;51(2):274-86.

9. Maccubbin D, Koren MJ, Davidson M, et al. Flushing profile of extended-release niacin/laropiprant versus gradually titrated niacin extended-release in patients with dyslipidemia with and without ischemic cardiovascular disease. Am J Cardiol. 2009;104(1):74-81.

10. Solish N, Bertucci V, Dansereau A, et al. A comprehensive approach to the recognition, diagnosis, and severity-based treatment of focal hyperhidrosis: recommendations of the Canadian Hyperhidrosis Advisory Committee. Dermatol Surg. 2007;33(8):908-23.

11. Moon DH, Kang DY, Kim DW, Kang MK, Lee S. Early results of new endoscopic thoracic sympathectomy for craniofacial hyperhidrosis. J Thorac Dis. 2018;10(6):3627-31.

12. Cardesín A, Alobid I, Benítez P, et al.
Barcelona Smell Test - 24 (BAST-24): Validation and smell characteristics in the healthy Spanish population. Rhinology. 2006;44(1):83-9.

13. Ware J, Kosinski M, Keller SD. A 12-Item Short-Form Health Survey: construction of scales and preliminary tests of reliability and validity. Med Care. 1996;34(3):220-33

14. Stangier U, Ehlers A, Gieler U. Measuring Adjustment to Chronic Skin Disorders: Validation of a Self-Report Measure. Psychol Assess. 2003;15(4):532-49.

15. Grolle M, Kupfer J, Brosig B, Niemeier V Hennighausen L, Gieler U. The skin satisfac tion questionnaire - An instrument to assess attitudes toward the skin in healthy persons and patients. Dermatology Psychosom. 2003;4(1):14-20.

16. Hilberg $O$, Pedersen OF. Acoustic rhinome try: recommendations for technical specifications and standard operating procedures. Rhinol Suppl. 2000;16:3-17

17. Deborah S, Prathibha KM. Measurement of Nasal Mucociliary Clearance. Clin Res Pulmonol. 2014;2(2):1019.

18. Anonymous. Methodologies to diagnose and monitor dry eye disease: report of the Diagnostic Methodology Subcommittee of the International Dry Eye WorkShop (2007). Ocul Surf. 2007:5(2):108-52.

19. Vadhanan P, Tripaty D, Adinarayanan S. Physiological and pharmacologic aspects of peripheral nerve blocks. J Anaesthesiol Clin Pharmacol. 2015;31(3):384.

20. George SMC, Atkinson LR, Farrant PBJ, Shergill BS. Botulinum toxin for focal hyperhidrosis of the face. $\mathrm{Br} J$ Dermatol. 2014;170(1):211-3.

21. Lee SH, Kim Y, Lim TY. Efficacy of sphenopalatine ganglion block in nasal mucosal headache presenting as facial pain. Cranio. 2018:8:1-3.

22. Eloy JA, Kovalerchik O, Bublik M, Ruiz JW, Casiano RR. Effect of greater palatine canal injection on estimated blood loss during endoscopic sinus surgery. Am J Otolaryngol - Head Neck Med Surg. 2014;35(1):1-4.

23. Neal JM, Gerancher JC, Hebl JR, et al. Upper extremity regional anesthesia: essentials of our current understanding. Reg Anesth Pain
Med. 2009;34(2):134-70

24. Tan G, Ma Y, Li H, Li W, Wang J. Long-term results of bilateral endoscopic vidian neurectomy in the management of moderate to severe persistent allergic rhinitis. Arch Otolaryngol - Head Neck Surg. 2012;138(5):492-7.

25. Horslen LC, Wilshire CL, Louie BE, Vallières $E$ Long-Term Impact of Endoscopic Thoracic Sympathectomy for Primary Palmar Hyperhidrosis. Ann Thorac Surg. 2018; 106(4):1008-1012

26. Glaser DA, Hebert AA, Pariser DM, Solish N. Facial hyperhidrosis: Best practice recommendations and special considerations. Cutis. 2007;79(5 Suppl):29-32.

27. Wilson TR, Birks Y, Alexander DJ. Pitfalls in the interpretation of standardised quality of life instruments for individual patients? A qualitative study in colorectal cancer. Qual Life Res. 2013;22(7):1879-88.

28. Glogau RG. Treatment of palmar hyperhidrosis with botulinum toxin. Semin Cutan Med Surg. 2001;20(2):101-8.

29. Bahmer FA. Quantification of sweat secretion in focal axillary hyperhidrosis related to area and time: the hyperhidrosis area and secretion index. Int J Dermatol. 2015;54(11):1233-5.

Eduardo Lehrer

Otolaryngology and Head and Neck

Surgery Department

Hospital Clínic de Barcelona

Universitat de Barcelona

Villarroel 170

08036 Barcelona, Catalonia

Spain

E-mail: elehrer@clinic.cat 